Shi-Jun Chen, Shi-Hui Li and Yan Zhao*

\title{
Crystal structure of catena-poly[triaqua-( $\mu_{2}-1,4$-di(pyridin-4-yl)benzene- $\left.\kappa^{2} N: N^{\prime}\right)$-(3',5-dicarboxy-[1,1'-biphenyl]-3,4'-dicarboxylato-kO)nickel(II)], $\mathrm{C}_{32} \mathrm{H}_{26} \mathrm{~N}_{2} \mathrm{O}_{11} \mathrm{Ni}$
}

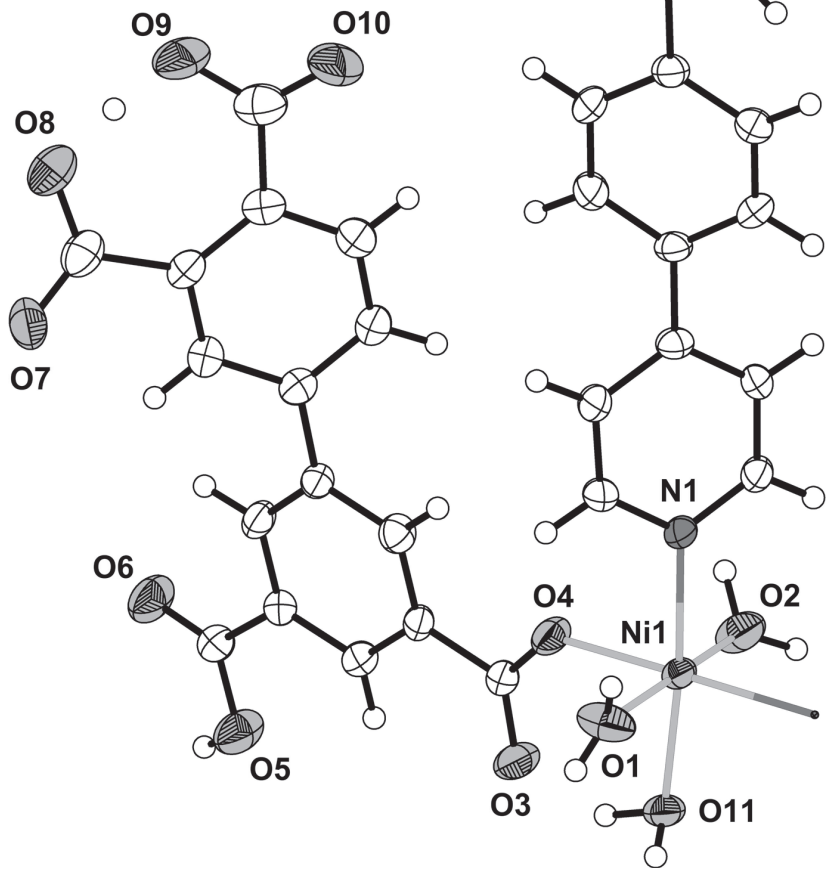

https://doi.org/10.1515/ncrs-2020-0196

Received April 22, 2020; accepted June 2, 2020; available online June 10, 2020

\section{Abstract}

$\mathrm{C}_{32} \mathrm{H}_{26} \mathrm{~N}_{2} \mathrm{O}_{11} \mathrm{Ni}$, monoclinic, $P 2_{1} / \mathrm{c}$ (no. 19), $a=7.6085(5) \AA$, $b=23.5084(14) \AA, \quad c=15.8052(9) \AA, \quad \beta=94.207(6)^{\circ}$,

\footnotetext{
*Corresponding author: Yan Zhao, Physics and Electronic Information College, Luoyang Normal University, Luoyang, Henan 471934, P.R. China, e-mail: luoyangchangchun@126.com. https://orcid.org/0000-0002-1723-7683

Shi-Jun Chen: College of Chemistry and Chemical Engineering, Xi'an SHIYOU University, Xi'an 710065, Shaanxi Province, P.R. China Shi-Hui Li: College of Chemistry and Chemical Engineering, Luoyang Normal University, Luoyang, Henan 471934, P.R. China
}

$V=2819.4(3) \AA^{3}, Z=4, R_{\mathrm{gt}}(F)=0.0571, w R_{\text {ref }}\left(F^{2}\right)=0.1285$, $T=293(2) \mathrm{K}$.

\section{CCDC no.: 2007098}

Table 1 contains crystallographic data and Table 2 contains the list of the atoms including atomic coordinates and displacement parameters.

Table 1: Data collection and handling.
Crystal:

Size:

Wavelength:

$\mu$ :

Diffractometer, scan mode:

$\theta_{\max }$, completeness:

$N(h k l)_{\text {measured }}, N(h k l)_{\text {unique }}, R_{\text {int }}$ :

Criterion for $l_{\text {obs }}, N(h k l)_{\text {gt }}$ :

$N(\text { param })_{\text {refined }}$ :

Programs:

\author{
Green block \\ $0.43 \times 0.35 \times 0.24 \mathrm{~mm}$ \\ Mo $K \alpha$ radiation (0.71073 $\AA$ ) \\ $0.76 \mathrm{~mm}^{-1}$ \\ SuperNova, $\omega$ \\ $25.5^{\circ}, 98 \%$ \\ $14931,5148,0.062$ \\ $I_{\text {obs }}>2 \sigma\left(I_{\text {obs }}\right), 3922$ \\ 420 \\ CrysAlis $^{\text {PRO }}[1]$, SHELX $[2,3]$
}

\section{Source of material}

A mixture of $\mathrm{Ni}(\mathrm{OAc}) 2 \cdot 4 \mathrm{H}_{2} \mathrm{O}(0.1 \mathrm{mmol}, 0.0248 \mathrm{~g}), 1,4-$ di(pyridin-4-yl)benzene ( $0.2 \mathrm{mmol}, 0.015 \mathrm{~g})$ and 3,3',4,5'biphenyl tetracarboxylic acid (H4btc) $(0.1 \mathrm{mmol}, 0.0330 \mathrm{~g})$, was dissolved in $10 \mathrm{~mL} \mathrm{H}_{2} \mathrm{O}$. The solution was heated in a $23 \mathrm{~mL}$ Teflon-lined autoclave under autogenous pressure at $383 \mathrm{~K}$ for five days. After cooling to room temperature crystals formed. The green crystals were collected.

\section{Experimental details}

Coordinates of hydrogen atoms were positioned according to geometric considerations.

\section{Comment}

In the last decades, a large number of mixed ligands metal-organic frameworks (MOFs) have been reported. Mixed ligands-MOFs can be constructed from metal ions and carboxylic acid and nitrogen-containing ligands. In most situations, $\mathrm{N}$-containing ligands are electrically neutral, while carboxylate ligands are negatively charged. Among organic aromatic multicarboxylates like the biphenyl-tetracates can yield predetermined networks and have been widely utilized 
Table 2: Fractional atomic coordinates and isotropic or equivalent isotropic displacement parameters $\left(\AA^{2}\right)$.

\begin{tabular}{|c|c|c|c|c|}
\hline Atom & $x$ & $y$ & $z$ & $U_{\text {iso }} * / U_{\text {eq }}$ \\
\hline $\mathrm{Ni1}$ & $0.82166(6)$ & $0.743933(18)$ & $0.43306(3)$ & $0.02666(17)$ \\
\hline N1 & $0.8342(4)$ & $0.67566(12)$ & $0.51877(17)$ & $0.0261(7)$ \\
\hline $\mathrm{N} 2$ & $1.1080(4)$ & $0.30843(12)$ & $0.97874(19)$ & $0.0306(7)$ \\
\hline 01 & $1.0830(4)$ & $0.72959(12)$ & $0.40339(18)$ & $0.0486(8)$ \\
\hline $\mathrm{H} 1 \mathrm{~A}$ & 1.1327 & 0.7398 & 0.3629 & $0.073^{*}$ \\
\hline $\mathrm{H} 1 \mathrm{~B}$ & 1.1612 & 0.7108 & 0.4426 & $0.073^{*}$ \\
\hline 02 & $0.5560(3)$ & $0.75826(11)$ & $0.44906(18)$ & $0.0440(7)$ \\
\hline $\mathrm{H} 2 \mathrm{~A}$ & 0.4820 & 0.7895 & 0.4652 & $0.066^{*}$ \\
\hline $\mathrm{H} 2 \mathrm{~B}$ & 0.4862 & 0.7263 & 0.4595 & $0.066^{*}$ \\
\hline 03 & $0.6574(4)$ & $0.73670(11)$ & $0.23195(17)$ & $0.0450(8)$ \\
\hline 04 & $0.7385(4)$ & $0.68465(10)$ & $0.34556(16)$ & $0.0374(7)$ \\
\hline 05 & $0.3512(4)$ & $0.63898(11)$ & $-0.01313(18)$ & $0.0498(8)$ \\
\hline $\mathrm{H} 5$ & 0.2919 & 0.6369 & -0.0585 & $0.075^{\star}$ \\
\hline 06 & $0.2957(4)$ & $0.54653(12)$ & $-0.01021(18)$ & $0.0493(8)$ \\
\hline 07 & $0.8218(4)$ & $0.34525(12)$ & $0.16437(17)$ & $0.0456(8)$ \\
\hline 08 & $0.7549(5)$ & $0.28274(13)$ & $0.2576(2)$ & $0.0749(11)$ \\
\hline $\mathrm{H} 8$ & $0.688(8)$ & $0.278(3)$ & $0.325(4)$ & $0.11(2)^{\star}$ \\
\hline 09 & $0.6674(4)$ & $0.27825(13)$ & $0.3991(2)$ & $0.0635(9)$ \\
\hline 010 & $0.6642(4)$ & $0.33570(13)$ & $0.5067(2)$ & $0.0583(9)$ \\
\hline 011 & $0.8097(3)$ & $0.80557(10)$ & $0.34008(15)$ & $0.0350(6)$ \\
\hline $\mathrm{H} 11 \mathrm{~A}$ & 0.9021 & 0.8205 & 0.3255 & $0.053^{\star}$ \\
\hline $\mathrm{H} 11 \mathrm{~B}$ & 0.7665 & 0.7892 & 0.2864 & $0.053^{*}$ \\
\hline $\mathrm{C} 1$ & $0.7739(5)$ & $0.67724(15)$ & $0.5960(2)$ & $0.0273(8)$ \\
\hline $\mathrm{H} 1$ & 0.7236 & 0.7109 & 0.6133 & $0.033^{*}$ \\
\hline $\mathrm{C} 2$ & $0.7816(5)$ & $0.63232(14)$ & $0.6511(2)$ & $0.0273(8)$ \\
\hline $\mathrm{H} 2$ & 0.7359 & 0.6360 & 0.7038 & $0.033^{*}$ \\
\hline $\mathrm{C} 3$ & $0.8574(4)$ & $0.58111(14)$ & $0.6289(2)$ & $0.0256(8)$ \\
\hline $\mathrm{C} 4$ & $0.9152(5)$ & $0.57915(15)$ & $0.5474(2)$ & $0.0351(10)$ \\
\hline $\mathrm{H} 4$ & 0.9633 & 0.5457 & 0.5277 & $0.042^{*}$ \\
\hline $\mathrm{C} 5$ & $0.9017(5)$ & $0.62614(15)$ & $0.4958(2)$ & $0.0328(9)$ \\
\hline $\mathrm{H} 5 \mathrm{~A}$ & 0.9419 & 0.6233 & 0.4418 & $0.039^{\star}$ \\
\hline C6 & $0.8807(4)$ & $0.53286(14)$ & $0.6886(2)$ & $0.0258(8)$ \\
\hline $\mathrm{C} 7$ & $0.9127(5)$ & $0.54280(15)$ & $0.7753(2)$ & $0.0307(9)$ \\
\hline $\mathrm{H} 7$ & 0.9076 & 0.5798 & 0.7956 & $0.037^{\star}$ \\
\hline $\mathrm{C} 8$ & $0.9516(5)$ & $0.49941(15)$ & $0.8312(2)$ & $0.0318(9)$ \\
\hline $\mathrm{H} 8 \mathrm{~A}$ & 0.9736 & 0.5077 & 0.8886 & $0.038^{*}$ \\
\hline C9 & $0.9587(5)$ & $0.44313(15)$ & $0.8042(2)$ & $0.0273(8)$ \\
\hline C10 & $0.9195(5)$ & $0.43294(15)$ & $0.7182(2)$ & $0.0334(9)$ \\
\hline $\mathrm{H} 10$ & 0.9183 & 0.3957 & 0.6983 & $0.040^{\star}$ \\
\hline C11 & $0.8823(5)$ & $0.47668(15)$ & $0.6616(2)$ & $0.0345(9)$ \\
\hline $\mathrm{H} 11$ & 0.8578 & 0.4684 & 0.6044 & $0.041^{\star}$ \\
\hline $\mathrm{C} 12$ & $1.0082(5)$ & $0.39656(14)$ & $0.8643(2)$ & $0.0255(8)$ \\
\hline C13 & $1.1129(5)$ & $0.40652(15)$ & $0.9389(2)$ & $0.0341(9)$ \\
\hline $\mathrm{H} 13$ & 1.1518 & 0.4432 & 0.9521 & $0.041^{\star}$ \\
\hline C14 & $1.1586(5)$ & $0.36242(16)$ & $0.9929(2)$ & $0.0344(9)$ \\
\hline $\mathrm{H} 14$ & 1.2287 & 0.3704 & 1.0421 & $0.041^{\star}$ \\
\hline C15 & $1.0092(5)$ & $0.29924(16)$ & $0.9070(3)$ & $0.0358(10)$ \\
\hline H15 & 0.9732 & 0.2621 & 0.8949 & $0.043^{\star}$ \\
\hline C16 & $0.9570(5)$ & $0.34108(16)$ & $0.8496(2)$ & $0.0343(9)$ \\
\hline $\mathrm{H} 16$ & 0.8869 & 0.3318 & 0.8009 & $0.041^{\star}$ \\
\hline $\mathrm{C} 17$ & $0.5957(5)$ & $0.63822(14)$ & $0.2290(2)$ & $0.0253(8)$ \\
\hline C18 & $0.6210(5)$ & $0.58638(15)$ & $0.2704(2)$ & $0.0295(9)$ \\
\hline $\mathrm{H} 18$ & 0.6755 & 0.5858 & 0.3251 & $0.035^{\star}$ \\
\hline C19 & $0.5670(4)$ & $0.53538(14)$ & $0.2324(2)$ & $0.0256(8)$ \\
\hline $\mathrm{C} 20$ & $0.4842(5)$ & $0.53725(15)$ & $0.1511(2)$ & $0.0285(9)$ \\
\hline
\end{tabular}

Table 2 (continued)

\begin{tabular}{lrrrr}
\hline Atom & $\boldsymbol{x}$ & $\boldsymbol{y}$ & $\boldsymbol{z}$ & $\boldsymbol{U}_{\text {iso }} \boldsymbol{U}_{\text {eq }}$ \\
\hline H20 & 0.4488 & 0.5035 & 0.1242 & $0.034^{*}$ \\
C21 & $0.4535(5)$ & $0.58883(15)$ & $0.1094(2)$ & $0.0264(8)$ \\
C22 & $0.5099(5)$ & $0.63900(15)$ & $0.1486(2)$ & $0.0283(9)$ \\
H22 & 0.4900 & 0.6735 & 0.1207 & $0.034^{*}$ \\
C23 & $0.5979(5)$ & $0.48148(15)$ & $0.2792(2)$ & $0.0284(8)$ \\
C24 & $0.5803(5)$ & $0.47910(16)$ & $0.3661(2)$ & $0.0343(9)$ \\
H24 & 0.5485 & 0.5117 & 0.3947 & $0.041^{*}$ \\
C25 & $0.6094(5)$ & $0.42937(16)$ & $0.4101(2)$ & $0.0361(10)$ \\
H25 & 0.5963 & 0.4290 & 0.4682 & $0.043^{*}$ \\
C26 & $0.6576(5)$ & $0.37951(16)$ & $0.3710(2)$ & $0.0320(9)$ \\
C27 & $0.6847(5)$ & $0.38116(15)$ & $0.2836(2)$ & $0.0293(9)$ \\
C28 & $0.6531(4)$ & $0.43184(15)$ & $0.2406(2)$ & $0.0298(9)$ \\
H28 & 0.6695 & 0.4329 & 0.1829 & $0.036^{*}$ \\
C29 & $0.6683(5)$ & $0.69107(15)$ & $0.2721(2)$ & $0.0286(9)$ \\
C30 & $0.6670(5)$ & $0.32859(19)$ & $0.4297(3)$ & $0.0447(11)$ \\
C31 & $0.7569(5)$ & $0.33366(17)$ & $0.2317(3)$ & $0.0397(10)$ \\
C32 & $0.3598(5)$ & $0.58786(16)$ & $0.0236(2)$ & $0.0300(9)$ \\
\hline
\end{tabular}

$[4,5]$. On the other hand, 1,4-di(pyridin-4-yl)benzene serves as bridging ligand $[6,7]$.

In the title complex, there is one $\mathrm{Ni}(\mathrm{II})$ ion, three coordinated water molecules, one H2btc ligand, and one 1,4-di (pyridin-4-yl)benzene ligand in the asymmetric unit. $\mathrm{Ni}(1)$ is six-coordinated with a distorted octahedral geometry by four $\mathrm{O}$ atoms from one H4btc and three coordinated water molecules, and two $\mathrm{N}$ atoms from two 1,4-di(pyridin-4-yl) benzene ligands from the axial positions (see the figure). The $\mathrm{Ni}-\mathrm{N}$ bond lengths are 2.098(3) $\AA$ and 2.102(3) $\AA$, whereas the $\mathrm{Ni}-\mathrm{O}$ ones are in the range of 2.031(2) to 2.103(3) $\AA$. The bond angles of $\mathrm{O}-\mathrm{Ni}-\mathrm{O}$ are in the range of $86.40(11)^{\circ}-174.12(11)^{\circ}$.

Acknowledgements: The authors thank the Key Scientific Research Project for Universities of Henan Province (No. (20A150028) and Science and Technology Research Project of Henan Province (no. 182102310909) for financial support.

\section{References}

1. Agilent Technologies: CrysAlis ${ }^{\mathrm{PRO}}$ Software system, version 1.171.35.15. Agilent Technologies UK Ltd, Oxford, UK (2011).

2. Sheldrick, G. M.: SHELXT - Integrated space-group and crystal-structure determination. Acta Crystallogr. A71 (2015) 3-8.

3. Sheldrick, G. M.: Crystal structure refinement with SHELXL. Acta Crystallogr. C71 (2015) 3-8.

4. Li, S. H.; Zhao, Y.: Synthesis and crystal structure of a new three-dimensional $\mathrm{Co}$ (II) coordination polymer based on 3,3', 4,5'-biphenyl tetracarboxylic acid and 4,4'-bipyridine. Inorg. Nano-Met. Chem. 47 (2017) 256-259. 
5. Li, S. H.; Zang, J.; Guo, J. B.: Synthesis and crystal structure of a new three-dimensional Ho(II) coordination polymer based on

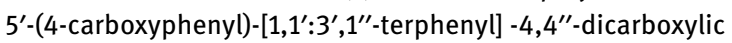
acid. Inorg. Nano-Met. Chem. 12 (2017) 1746-1749.

6. Li, D. M.; Li, S. H.: Crystal structure of 4,4'-(1,4-phenylene) bis(pyridin-1-ium) catena-poly[diaqua-bis $\left(\mu_{2}-3^{\prime}, 5^{\prime}\right.$-dicarboxy- [1,1'-biphenyl]-2,5-dicarboxylato- $\left.\mathrm{K}^{2} \mathrm{O}: \mathrm{O}^{\prime}\right]$ dihydrate. Z. Kristallogr. NCS 232 (2017) 971-973.

7. Song, W.; Xu, X. L.; Yang, Y. J.; Li, S. H.: Synthesis and crystal structure of catena-poly[bis(2-(2-((2,6-dichlorophenyl) amino)phenyl)acetato-k $\left.O ; \mathrm{k}^{2} O^{\prime}, O^{\prime \prime}\right)-\left(\mathrm{k}^{2}-1,4-\mathrm{di}(\right.$ pyridin-4-yl) benzene- $\left.{ }^{2} N: N^{\prime}\right)$ zinc(II)]. Z. Kristallogr. NCS 233 (2018) 399-401. 